\title{
Mapeamento de softwares de gestão da produção: análise voltada para as micro e pequenas empresas
}

\author{
Luís Cláudio Figueira Mendes \\ Júnior \\ luisclaudio.007@hotmail.com \\ Universidade do Estado do Pará (UEPA), \\ Castanhal, Pará, Brasil \\ Denilson Ricardo de Lucena \\ Nunes \\ denilson.lucena@gmail.com \\ Universidade do Estado do Pará (UEPA) \\ Castanhal, Pará, Brasil \\ Léony Luís Lopes Negrão \\ leony@uepa.br \\ Universidade do Estado do Pará (UEPA), \\ Castanhal, Pará, Brasil \\ André Cristiano Silva Melo \\ acsmelo@yahoo.com.br \\ Universidade do Estado do Pará (UEPA), \\ Universidade do Es
Belém, Pará, Brasil \\ Elida Roberta Carvalho Xavier \\ elidakarvalho13@gmail.com \\ Universidade do Estado do Pará (UEPA), \\ Castanhal, Pará, Brasil
}

\begin{abstract}
RESUMO
O objetivo desta pesquisa foi obter um levantamento de informações que pudessem apresentar à comunidade acadêmica e profissional de gestão de softwares sobre as modalidades livre, gratuito e proprietário, bem como suas principais funcionalidades, baseando-se nas abordagens MRPI, MRPII e ERP. A pesquisa reúne conceitos sobre Planejamento e Controle da Produção, Sistemas de Administração da Produção, software livre, gratuito e proprietário, vantagens e desvantagens na utilização desses softwares, além de informações sobre micro e pequenas empresas e suas particularidades. As informações coletadas forma reunidas em um mapeamento que foi divido em três aspectos básicos: viabilidade de instalação, funcionalidades e usabilidade, mesmos aspectos utilizados no estudo de Campos (2006). O estudo foi realizado através de uma revisão da literatura qualitativo-positivista, processo metodológico descritivo no qual foi realizada a análise heurística e seleção. Para o mapeamento dos softwares livres foi utilizado o site SourceForge.net, para os softwares gratuitos utilizou-se sites afins e para o proprietário sites como Abril, UOL e outros. Os resultados apontaram que existe uma infinidade maior de softwares ERP em detrimento dos MRPI e MRPII, mostram ainda as características gerais dos softwares mapeados e outras informações detalhadas.
\end{abstract}

PALAVRAS-CHAVE: Sistemas de administração da produção. Software livre. Software gratuito. Software proprietário. Micro e pequena empresa. 


\section{INTRODUÇÃO}

De acordo com Sebrae (2014), as e Pequenas Empresas (MPE's) empregavam em torno de $52 \%$ de toda mão de obra formal do país, por conseguinte os salários pagos pelas mesmas correspondem a $40 \%$ da massa salarial brasileira, isto é além de admitir mais da metade dos brasileiros, também movimenta em salários aos trabalhadores aproximadamente $50 \%$ dos pagamentos formais do país.

O Governo Federal anunciou a liberação de $\mathrm{R} \$ \$$ 8,2 bilhões em financiamentos para pequenas e microempresas para os próximos 2 anos a começar por março de acordo com G1 (2017), análogo fator pode ser capaz de induzir essas empresas a um aperfeiçoamento nos seus sistemas produtivos, tais como a aquisição de softwares para ajudá-las na tomada de decisões.

No momento em que se fala da aplicação de certos tipos de softwares nas MPE's que deem uma melhor gestão a suas atividades frente à concorrência, dentro de um ambiente turbulento na qual estão inseridas.

De acordo com Profissionais TI (2014), as pequenas empresas buscam utilizar da tecnologia da informação para satisfazer melhor seus clientes, podendo assim conquistar ganhos sobre a concorrência, do mesmo jeito que as mesmas coletam e usam da informação acessível para administrar suas atividades de maneira mais efetiva.

A tecnologia está disponível e pode auxiliar a aumentar a produtividade, administrar estoques, movimentos financeiros, apontar necessidades e preferências de clientes e ainda prever dificuldades e oportunidades.

Porém, apesar da grande funcionalidade dos softwares na gestão de informações, a dificuldade está em selecionar aquele que seja mais adequado à realidade de cada empresa (seja no custo, na robustez ou complexidade), pois na prática muitas vezes as empresas não conhecem as opções disponíveis no mercado. $\mathrm{O}$ alcance dos softwares desenvolvidos com o modelo livre, gratuito e proprietário têm atingido áreas distintas: sistemas gerenciadores de banco de dados, servidores de página web, linguagens de programação de amplo nível, administração da produção e o planejamento e controle da mesma.

As empresas tendem a se concentrar em aspectos como custo, robustez, autonomia da tecnologia, provedor, além de capacidade de personalização dos softwares disponíveis no mercado. Posto isto, precisam observar diversos desses softwares e identificar qual se adequa melhor às suas necessidades (SEBRAE,2016).

Segundo Sebrae (2014), enquanto as grandes empresas apoiam-se em softwares para melhorar a administração, as pequenas ainda buscam fundamentos que Ihes ajudem a desenvolver o básico da produção, medir o resultado do trabalho e avaliar seu desempenho.

Com isso o presente trabalho visa auxiliar as micro e pequenas empresas (MPE's) na busca por softwares voltados as filosofias MRP I, MRPII e ERP, desenvolvidos sobre os modelos livre, gratuito e proprietário. 


\section{REVISÃO DA LITERATURA}

\section{PLANEJAMENTO E CONTROLE DA PRODUÇÃO (PCP)}

O planejamento e controle de produção é um sistema que comanda tarefas de produção, reunindo informações que depois de organizadas serão distribuídas aos setores envolvidos.

Segundo Slack et al. (2015), um princípio da administração da produção é que o Planejamento e Controle da Produção (PCP) abrange tanto programar, coordenar as atividades sendo um sistema que chefia tarefas de produção, reunindo informações que após organizadas, podem ser distribuídas aos setores envolvidos, tentando conciliar assim as demandas existentes no mercado e a competência dos suprimentos de produção para entregá-las.

Perante a competitividade, as empresas buscam se adequar constantemente, procurando melhorias contínuas em sua produtividade. Para tanto, a utilização de sistemas flexíveis e que integram toda a administração é determinante. Dentre esses se destaca o PCP.

No conjunto de utilidades e atividades desenvolvidas pelo PCP estão o delineamento de objetivos e técnicas, criação de planos, controle dos recursos humanos e físicos, e orientação para o ajustamento de possíveis desvios, compreendendo planos realizados nos âmbitos estratégicos, tático e operacional (TUBINO, 2009).

Com o objetivo de operacionalizar e dirigir o PCP, organizando a preparação dos dados e a tomada de decisões, as organizações criam um setor, ou seção, de auxílio à produção, geralmente agregado à gerência industrial, conhecido como PCP (Seção de Planejamento e Controle da Produção), ou, em algumas empresas, PPCP (Seção de Planejamento, Programação e Controle da Produção). Essa seção de ajuda possui como responsabilidade apontar e adaptar os recursos produtivos, a fim de reparar com efetividade os planos determinados nos níveis estratégico, tático e operacional (TUBINO, 2009).

Além disso, o PCP envolve todos os níveis hierárquicos e em cada um deles assume um papel diferenciado. São delineados planejamentos específicos para cada nível, constantemente procurando adquirir o propósito maior, no qual são estabelecidas as atividades a serem desenvolvidas (MARTINS \& LAUGENI,2015), em níveis estratégico, tático e operacional (Tubino, 2009).

No nível estratégico é definida a Estratégia de Produção, gerando um Plano de Produção. No campo tático, são estabelecidos planos de médio prazo, o PlanoMestre de Produção (PMP), através do Planejamento-Mestre da Produção (Tubino, 2009). Finalmente, ao nível operacional, no qual são definidos os programas de curto prazo pertinentes e realizados programas de acompanhamento destes, prepara-se a Programação da Produção, coordenando estoques, dando Ordens de Compras, Fabricação e Montagem, bem como há o Acompanhamento e Controle da Produção, gerando um relatório de Avaliação de Desempenho (MARTINS \& LAUGENI, 2015).

O controle permite realizar ajustes para que a operação alcance aquilo que o planejamento estabeleceu, é de alto valor que as organizações, tanto grandes como pequenas, saibam essas diferenças, pois assim diminuem os riscos de atropelarem etapas no seu processo produtivo (SLACK et al, 2015). 


\section{SISTEMAS DE ADMINISTRAÇÃO DA PRODUÇÃO (SAP)}

Os Sistemas de Administração da Produção (SAP) são o núcleo dos processos produtivos. Eles têm o objetivo básico de planejar e controlar o processo de manufatura em todos seus níveis, incluindo materiais, equipamentos, pessoas, fornecedores e distribuidores (SLACK et al., 2015).

É através dos SAP que a organização garante que suas decisões operacionais sobre o que, quando, quanto, com o que produzir, com quem comprar seja adequado às suas necessidades estratégicas que, por sua vez, são ditadas por seus objetivos e seu mercado (TUBINO,2009).

\section{MRP - Material Requirement Planning}

Segundo Slack et al. (2015), o MRP é um sistema que calcula a quantidade de materiais que será necessária e para quando será necessário, utilizando para esses cálculos, os pedidos em carteira e uma previsão de demanda. Este por sua vez verifica todos os ingredientes ou componentes que serão necessários para completar os pedidos, garantido assim a quantidade correta no tempo correto.

Segundo Martins \& Laugeni (2015), os elementos compostos por esse sistema são:

- Lista de material (BOM) - Faz-se aqui a listagem dos materiais necessários para a fabricação de produtos;

- Controle de estoque - Mostra-se a quantidade de materiais que estão disponíveis em estoque na organização, e tal controle é importante, pois o MRP poderá buscar alternativas tais como estoque de segurança com intuito de absorver eventuais ocorrências não previstas;

- Plano mestre - Este plano nos mostra a demanda a ser atendida, já depurada dos fatores externos, isto é, aquilo que deve ser realmente produzido. Em outras palavras é o programa de produção elaborado pelo PCP, com suas respectivas quantidades e prazos acordados com o cliente, envolvendo também a programação de recursos físicos (máquinas) e humanos (mão de obra);

- Compras - O MRP gera uma lista de materiais a serem comprados, onde o setor de compras deve atuar.

Existem várias empresas que têm seus sistemas interligados junto a fornecedores parceiros e assim os pedidos de reabastecimento poderão ser feitos diretamente pelo computador;

Para que o PMP (Plano Mestre de Produção) possa realizar seus levantamentos como: o volume e a ordem de montagens, submontagem e materiais necessários, faz-se necessário a criação de uma lista de materiais (LDM) que será o levantamento das partes requeridas para a fabricação dos produtos.

Observa-se na Figura 1, uma abrangência do que os sistemas MRP I necessitam e sua ligação intrínseca com o PMP. 
Figura 1 - Visão geral do sistema MRP I

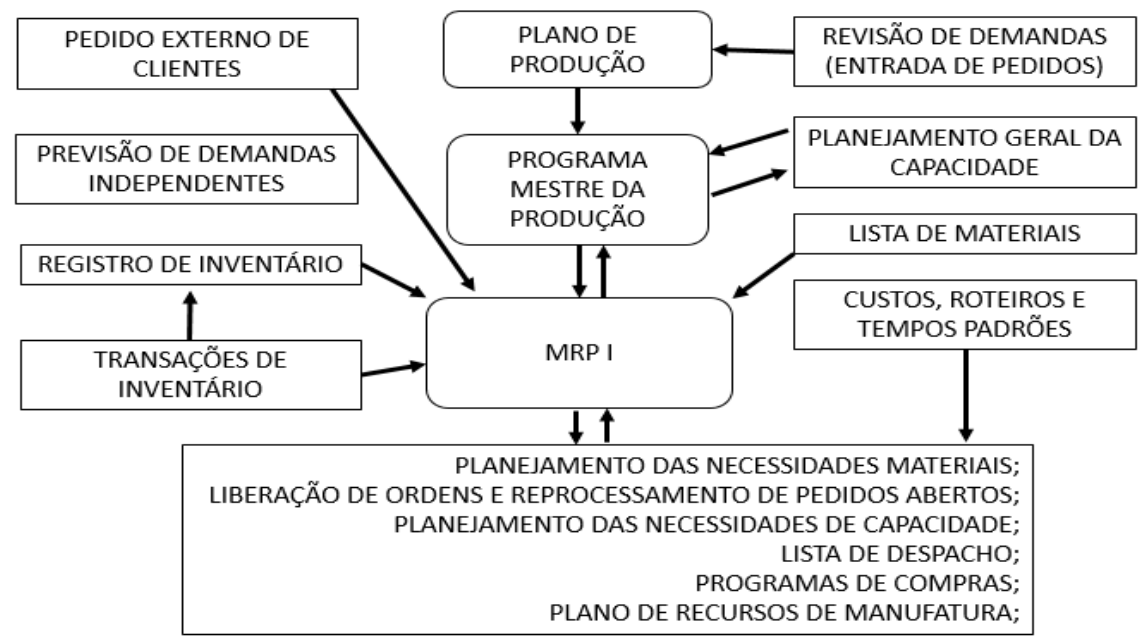

Fonte: Adaptado de Correa et al. (2001)

\section{MRP II - Manufaturing Resource Planning}

Martins \& Laugeni (2015) ressaltam ainda que, durante os anos 80 e 90, o sistema e o conceito do MRP expandiram-se e foram integrados a outras partes da organização. Essa versão ampliada do MRP é conhecida atualmente como Planejamento dos Recursos de Manufatura (Manufacturing Resource Planning) ou MRP II.

O MRP II nada mais é que um sistema que contribui para o desenvolvimento eficaz da administração da produção, quando os planos de longo prazo de produção, se tornam conjuntos que agregam níveis iguais de trabalho por todo o mundo, inclusive setores produtivos, sendo surpreendentemente acurado até se alcançar ao nível do planejamento de componentes e máquinas especificas e desejadas (CORRÊA E CORRÊA, 2011).

BOCKERSTETTE e SHELL (1993) complementam dizendo que "O MRP II é um sistema de planejamento e controle administrado por computador que se utiliza do "pull system" para empurrar a fabricação, permitindo a não formação de exageros de especificação no processamento produtivo".

O MRP II tem cinco módulos principais: módulo de planejamento, módulo de planejamento mestre de produção, módulo de cálculo de necessidade de materiais, módulo de cálculo de necessidade de capacidade e módulo de controle de indústria. Há também, alguns módulos de atualização de dados cadastrais, que tratam de cuidar das modificações quanto aos dados de produtos de um reserva, demais estruturas de produtos, centros produtivos, roteiros de produção, dentre mais (CORREA E CORREA,2011).

São inúmeras empresas que usam e estão programando a implantação do MRP II em seus serviços. Um dos elementos essenciais que colaboram para a efetivação desse processo é coletar dados e informações corretas de uma empresa, como um raio " $x$ " completo (SLACK et al. ,2015).

$\mathrm{O}$ refinamento dos dados brutos e um MPS efetivo constroem um caminho para uma gestão de sucesso, acompanhado de uma listagem de materiais, que conseguirá ser usada como manual para eventuais necessidades. Também contribuem para esse fim o registro completo de estoques adaptados, o apoio e 
participação do lado gerencial da empresa, treinamentos adequados, planos de efetivação claros e metas, e uma equipe qualificada e responsável (SLACK et al. ,2015).

Tendo tudo isto em mãos, a operacionalização depende de uma série de elementos, tais como: disponibilidade de mão de obra, conflitos entre o sistema antecedente e o atual, complexidade da base de dados, complexidade da linha de produção, e nível de advento de complicações do formato antecedente. Para entender de que maneira deve-se começar a implantação do MRP II, pode-se definir a maneira de efetivação do MRP II em 3 formas segundo (BOCKERSTETTE e SHELL, 1993).

Analisando a Figura 2, pode-se observar que o MRP permite que as empresas calculem quantos e que tipo de material serão necessários ao processo produtivo e em que momento. Para isso, o MRP utiliza-se de informações como os pedidos realizados e a previsão de pedidos futuros. O MRP II permite que as empresas avaliem as implicações de demandas futuras, assim como analisam as implicações de como gerir os recursos com relação à necessidade de materiais.

Figura 2- Análise do Sistema MRPII

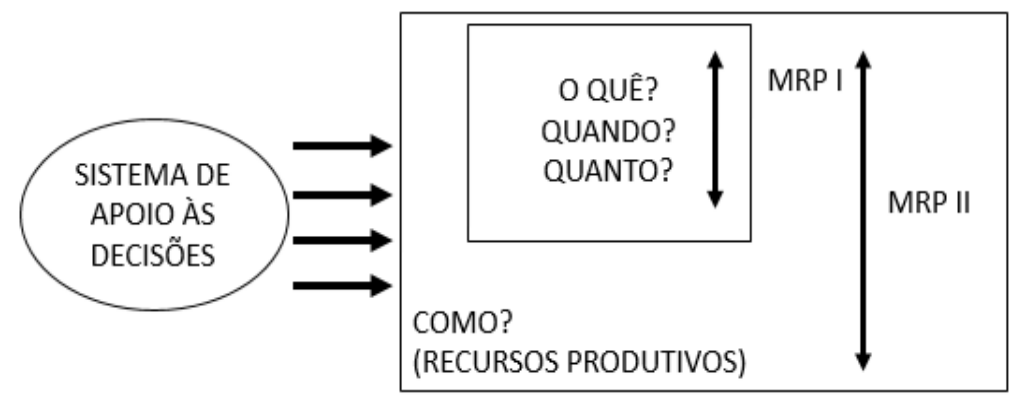

Fonte: Corrêa et al. (2001)

\section{ERP - Enterprise Resource Planning}

De acordo com Slack et al. (2015), o surgimento do ERP como sistema de apoio à produção se deu a partir do MRPII que teve sua expansão durante a década de 80. A inovação tecnológica permitiu tal avanço desde o melhoramento de internet, computadores, melhor processamento e comunicação dentro das organizações. Foi possível também a prática de modelagem de cenários futuros como a pergunta "o que ocorreria se...", e os dois sistemas tratados nos tópicos anteriores trabalham com essa temática de fazer as adaptações necessárias para mudanças de operação, o ERP trabalha com isso de forma mais ampla como é definido abaixo:

[...] é um software de planejamento dos recursos empresariais que integra as diferentes funções da empresa, para criar operações mais eficientes. Integra os dados-chave e a comunicação entre as áreas da empresa, fornecendo informações detalhadas sobre as operações da mesma (POZZO, 2009).

Os benefícios dos softwares ERP são inúmeros, como os apontados por Slack et al. (2015): softwares comunicação bem mais sofisticados com fornecedores, clientes e outros parceiros comerciais integrados, rotineiramente fornecendo informações mais precisas, além de ser capaz de integrar redes de suprimentos completas, incluindo fornecedores de fornecedores e clientes de clientes. 
De acordo com Martins \& Laugeni (2015) as características e benefícios do ERP são de difícil mensuração pelas organizações, visto que a integração com todas as partes pode se tornar algo extremamente caro, por conta das mudanças necessárias, quando os sistemas antigos com a integração se tornam irrelevantes. Algumas vezes até a própria cultura organizacional da organização impõe barreiras que também podem dificultar a adaptação a esse novo tipo de sistema.

Aconselha-se fazer a substituição aos poucos na organização, com novos sistemas comuns e com banco de dados que se relacionam com melhor eficiência e que possam ajudar a assegurar a transferência de dados entre os diferentes setores das empresas (MARTINS \& LAUGENI,2015).

Os sistemas ERPs se fortaleceram no ambiente empresarial a partir da integração da gestão de diferentes funções do negócio, aprimorando o desempenho dos processos como um todo. O ERP é considerado a evolução do MRP II, como ilustrado na Figura 3, mostrando assim o quanto integrado pode ser esse tipo de sistema.

Figura 3- Evolução estrutural dos sistemas ERP

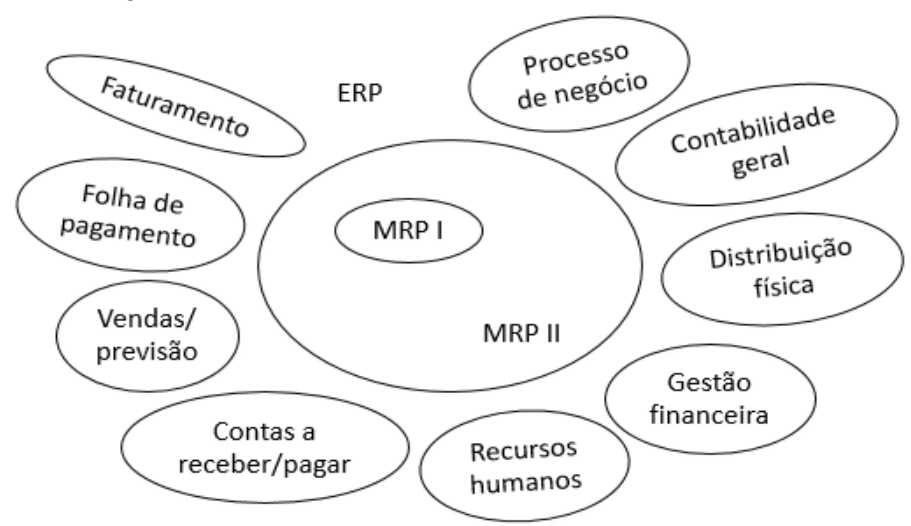

Fonte: Adaptado de Corrêa et al.(2011)

\section{SOFTWARE LIVRE, GRATUITO E PROPRIETÁRIO}

Nessa seção serão apresentadas as características dos 3 tipos de softwares levantados no presente trabalho.

\section{Software livre}

Pode-se considerar que é um software que respeita a liberdade e sensatez de coletividade dos usuários, significando que os usuários adquirem a liberdade de cumprir, arremedar, racionar, estudar, desviar e aumentar o software. Em vista disso, é uma ideia de liberdade, não de preço. Com essas liberdades, os usuários (tanto individualmente quanto coletivamente) controlam o programa e o que ele faz por eles (FREE SOFTWARE FOUNDATION, 2017).

De acordo com Silveira (2004, pág. 9) "As quatro liberdades que caracterizam o software livre são as de consumo, abastamento, modificações e redistribuição". De maneira mais concreta, Reis (2003, p. 14) define software livre como: 
...qualquer aplicativo cujo uso garanta ao seu cliente liberdades relacionadas ao consumo, alteração e redistribuição. Seu enfoque essencial é o fato do código-fonte estar livremente disponível para ser entendido, estudado ou alterado por qualquer pessoa interessada.

As vantagens deste tipo de software é que são capazes de ter seu códigofonte modificado por qualquer cliente; não necessita de licença para distribuição. Atualizações constantes, mantendo o sistema estável e sem riscos, abrangem o ofício de desenvolver aplicações e design de softwares populares, como o Windows e o pacote Microsoft Office, para se tornarem familiares aos usuários. Interfaces amigáveis tornam esses softwares capazes de serem utilizados em desktops, com liberdade para explorar a linguagem e adaptá-la às necessidades, na administração de redes (SEBRAE,2016).

As desvantagens são que costumam ter interfaces pouco intuitivas, as instalações são mais complexas, problemas com estabilidade, e há pouca variedade nos programas compatíveis com o sistema operacional, além de serem pouco conhecidos. Somente pessoas com treinamento avançado "dominam" o Software Livre e a manutenção desses sistemas é mais complexa e demanda mão de obra qualificada, consequentemente com custos mais elevados (SEBRAE,2016).

\section{Software gratuito}

Um software gratuito é aquele no qual o programa pode ser copiado e distribuído gratuitamente. Essa forma de aplicativo é fornecida apenas em sua maneira binária, isto é, com o programa executável. O código fonte, que são as operações que formam o programa, não é disponibilizado, o que significa que ele não pode ser alterado, e nem sequer examinado. O programa apenas pode ser utilizado da maneira como foi disponibilizado, e consequentemente existem limitações na sua distribuição (CANALTECH).

Estes tipos de softwares são vantajosos apenas por serem gratuitos, a desvantagem é que a maior parte têm funcionalidades limitadas com o intuito de induzir o cliente a buscar as versões pagas para usar todas as funcionalidades do software. Além disso, a maior parte dos softwares gratuitos disponibilizados na internet costuma direcionar para a instalação de outros softwares indesejáveis, maliciosos e potencialmente perigosos, além do suporte e preservação serem limitados (CANALTECH).

\section{Software proprietário}

No momento em que se fala em software proprietário está se falando de um modelo de progresso e abastecimento alicerçado em licenças restritivas de consumo, ou seja, em autoria e propriedade de software. As pessoas estariam impedidas de adulterar e redistribuir o mesmo (SILVEIRA, 2004).

Ao contrário do software livre, software proprietário é aquele cuja cópia, redistribuição ou modificação é proibida pelo seu autor ou distribuidor. Geralmente para utilizar ou ter acesso ao código-fonte ou redistribuir, deve-se 
pedir autorização ao proprietário ou pagar para fazê-lo. Em outras palavras, é necessário ter a licença do produto (CANALTECH).

A vantagem destes softwares é que apresentam maior agilidade, são adaptáveis com a maior parte dos sistemas operacionais conhecidos, é são mais populares entre os usuários, fazendo com que se sejam fácil acesso, além de suporte técnico e manutenção (CANALTECH).

As desvantagens é que são pagos e licenciados, isto é, não se pode adaptá-lo conforme as necessidades, o que pode causar alguns prejuízos às companhias que o utilizam por frequentes paralisações ou perdas de dados (CANALTECH).

\section{MICRO E PEQUENAS EMPRESAS E TECNOLOGIA DE INFORMAÇÃO}

Para o estado, a classificação para micro e pequenas empresas é baseada na receita bruta anual igual ou inferior a $\mathrm{R} \$ 360.000,00$. As microempresas representam uma comunidade empresária, empresa individual de responsabilidade limitada (SEBRAE,2016).

Caracterizam-se por executar atividades com baixa necessidade de capital e com alta intensidade de mão de obra. $O$ pequeno empresário, nos períodos de pouco movimento, supera as dificuldades ele próprio "pegando no pesado" e, várias vezes, recruta de familiares para Ihe auxiliar (GUARDA,2003).

A tecnologia da Informação (TI) nas Micro e Pequenas Empresas (MPE's) amplifica seu potencial quanto à utilização da informação, como um recurso estratégico, uma vez que ela traz modificações no espaço e no tempo (BERALDI, 2002).

Assim, a pequena empresa que tem um sistema informatizado funcionando de maneira eficiente obtém benefícios, como o tempo otimizado, a aquisição de informações, projeções futuras mais precisas, dentre outros aspectos, que contribuem para o sucesso da organização. A informatização das MPE's aumenta sua competitividade e também o lucro (BERALDI e FILHO, 2000). Ela é de grande valia para a conservação das MPE's, por ser um instrumento com capacidade de sustentar sua competitividade, porém devido às limitações financeiras das MPE's e inclusive com a postura de algumas empresas, especializadas em $\mathrm{TI}$, em fazer produtos e serviços voltados para as MPE's, também nem sequer todos os recursos de TI são acessíveis a esse segmento (BERALDI, 2002).

De acordo com Moraes (2005), isso se dá pela dificuldade de aceitação dos produtos disponíveis no mercado, as formas de gestão das pequenas empresas. Também para o mesmo autor, esse feito acontece devido às pesquisas e ao progresso em TI estar voltado para as grandes organizações, no qual sua plataforma não é flexível o suficiente para aplicações em organizações menores.

Há obstáculos que são tidos como os principais entraves ao consumo de TI nas MPE's apresentados por Moraes (2005):

1. Custos, frequentemente elevados, que exigem grandes investimentos frente aos seus limitados recursos financeiros;

2. A maior parte das pesquisas é realizada em grandes empresas, envolvendo suas realidades, não apropriadamente aplicáveis nos ambientes das pequenas empresas; 
3. As pequenas empresas possuem dificuldades em aderir a softwares disponíveis no mercado, uma vez que são complexos demais para seu ambiente específico;

4. Ao absorver a tecnologia, faz de forma lenta;

5. Dificuldade em alimentar o sistema com informações corretamente;

6. Forte resistência por parte dos funcionários que entendem que serão superados pela tecnologia;

7. Falta de tempo do proprietário dirigente para se inteirar do processo de implantação de novas tecnologias, bem como para realizar um bom treinamento.

\section{Mapeamento utilizado em Campos (2006) para softwares nas MPE's}

Através da revisão da literatura levantada no presente trabalho e de Campos (2006), foi possível observar as perguntas utilizadas para fazer o mapeamento dos softwares, assim como a metodologia utilizada para a melhor filtragem dos softwares.

Para o mapeamento dos softwares livres, similar ao trabalho de Campos (2006), no presente trabalho também foi utilizado o repositório de bancos de dados chamado SourceForge, não só pelo fato de ser um pioneiro em fornecer o serviço de hospedagem de projetos, conforme declara Reis (2003), mas também por ele ter sido base para outros trabalhos, envolvendo projetos de software livre, por exemplo, krishnamurthy (2002) utilizou como fonte para um artigo intitulado Cave or comumunity? Na Empirical Examination of 100 Mature Open Souce Projects.

O site SourceForge.net é um localizador centralizado de desenvolvedores de software para controlar e manter o desenvolvimento de open sources e atua como um repositório de código fonte (TOSTES,2009). Segundo Campos (2006), em maio de 2005, o site registrava 110.650 projetos cadastrados, em 2018 já registra mais de 360.000 projetos cadastrados, segundo o próprio site, números que mostram que constantemente, há maior absorção de seguidores e idealizadores de softwares livres.

Campos (2006) mapeou os softwares ERP na modalidade livre e seu trabalho buscou o mapeamento além dos softwares livres, como também as modalidades proprietário e gratuito, assim como os softwares MRP I e MRP II, para isso utilizase do Anexo A e B de Campos (2006), para a resposta dos dados coletados dos softwares, sendo feitas adaptações e a criação de tabelas que foram evidenciadas no Anexo C.

Para os softwares MRP I e MRP II sobre a modalidade livre utilizou-se o mesmo site sourceforge.net, pois o mesmo apresenta esses tipos de softwares cadastrados, para os softwares MRP I, MRP II e ERP na modalidade gratuito e proprietário, como não se encontrou um site especifico que trabalhasse com essas modalidades, buscou-se sites como o da UOL, Abril e sites afins, para guiar no mapeamento desses softwares.

\section{METODOLOGIA}

A metodologia desse trabalho se apresenta como descritiva pelo fato de buscar quais são os softwares que trabalham com MRP, MRP II e ERP 
desenvolvidos de forma livre, gratuita e proprietária, que poderiam ajudar as micro e pequenas empresas. O delineamento do estudo foi indicado como o "Levantamento", ou seja, a procura dos softwares MRP I, MRP II e ERP na modalidade livre a partir do site SourceForge, assim como Campos (2006). Nas modalidades, gratuito e proprietário a busca ocorreu via sites como o UOL, Abril e sites dos próprios desenvolvedores dos softwares.

Para a coleta, seleção e caracterização dos softwares identificados, foram analisadas as mesmas questões usadas por Campos (2006) que deram origem a um grupo de questões adaptadas pelos autores desta pesquisa (conforme Quadros 1 e 2). As questões observadas em Campos (2006) foram do tipo: "Há a necessidade de instalação de software adicional para que o aplicativo possa funcionar adequadamente?" "Se há, quais são eles?", "O software tem um programa instalador que auxilie no processo de instalação?", dentre outras, a partir destas questões foram propostos os Quadros 1 e 2, para a caracterização dos softwares que foram pré-selecionados como sendo em língua portuguesa e que fosse voltado para o suporte do PCP, a partir das abordagens MRPI, MRPII e ERP. A abordagem utilizada nesta pesquisa, segundo Barcelos (2001), pode ser fácil classificada como qualitativa.

Quadros 1 - Quesitos observados nos softwares proprietários Softwares Proprietários

\begin{tabular}{ll}
\hline Nome: & \multicolumn{1}{l}{ Tipo: } \\
\hline Web site: & Possui Demo: \\
Sistema Operacional: & Taxa única: \\
Mensalidade: & Teste grátis por 30 dia: \\
Indicação Hardware: & Opçães de desinstalação: \\
Possui instalador: & Possui relatórios: \\
Dificuldade de instalação: & Gerador de gráficos: \\
Software adicional: & Acesso a mais usuários: \\
Número de Download: & Interface natural: \\
Idioma: & Mecanismo detecc̃a de erros: \\
Gerencia mais de uma empresa: & Objetos e ações fáceis e visíveis: \\
Exporta relatórios em arquivo: & Solução,dúvidas, utilização: \\
Possibilita integração com outro software: & Suporte: \\
Senha para acesso: & Avaliação do Autor: \\
Backup interno: & Avaliação dos Usuários: \\
Expressões habituais usuário: & \\
Indicadores de resultados e controles: & \\
Mensagens clara de erros: & \\
Documentação disponível: & \\
\hline
\end{tabular}

Fonte: Autores (2018, adaptado de Campos, 2006)

\section{RESULTADOS E ANÁLISE DOS DADOS}

Esta seção contém os resultados a partir dos softwares que foram mapeados via no site sourceForge.net, UOL, Abril, entre outros, onde foram detalhadas as características de cada um deles, baseadas nos Quadros 1 e 2. Inicialmente no 
Quadro 3 são apresentados os softwares selecionados conforme descrito na seção de metodologia.

Quadro 2-Quesitos observados nos softwares livres e gratuitos

Softwares Livres e Gratuitos

\begin{tabular}{|c|c|}
\hline Nome: & Tipo: \\
\hline \multicolumn{2}{|l|}{ Web site: } \\
\hline Sistema Operacional: & Possui Demo: \\
\hline \multicolumn{2}{|l|}{ Indicação Hardware: } \\
\hline Possui instalador: & Opções de desinstalação \\
\hline Dificuldade de instalação: & Possui relatórios: \\
\hline Software adicional: & Gerador de gráficos: \\
\hline Número de Download: & Mecanismo detecção de erros: \\
\hline Idioma: & Acesso a mais usuários: \\
\hline Gerencia mais de uma empresa: & Interface natural: \\
\hline Exporta relatórios em arquivo: & Objetos e ações fáceis e visíveis: \\
\hline $\begin{array}{l}\text { Possibilita integração com outro software: } \\
\text { Senha para acesso: }\end{array}$ & $\begin{array}{l}\text { Solução,dúvidas, utilização: } \\
\text { Suporte: }\end{array}$ \\
\hline Backup interno: & Avaliação do Autor: \\
\hline Expressões habituais usuário: & Avaliação dos Usuários: \\
\hline \multicolumn{2}{|l|}{ Indicadores de resultados e controles: } \\
\hline \multicolumn{2}{|l|}{ Mensagens clara de erros: } \\
\hline \multicolumn{2}{|l|}{ Documentação disponível: } \\
\hline
\end{tabular}

Fonte: Autores (2018, adaptado de Campos, 2006)

Quadro 3-Softwares identificados pela pesquisa

\begin{tabular}{|c|c|c|c|c|}
\hline \multirow{4}{*}{ Gratuito } & Marketup & $\begin{array}{c}\text { ERP Lite Free } \\
\text { Plus }\end{array}$ & Bitrix24 Free & Mercado Backoffice \\
\hline \multirow{4}{*}{ Livre } & Fibiese & Freedom ERP & OpenXpertya & OpenERP \\
\cline { 2 - 5 } & Compiere & Dolibarr ERP & Adempierre ERP & Vtiger CRM \\
\cline { 2 - 5 } & WebERP & Open22ERP & ERP CRM & Openbravo \\
\hline \multirow{4}{*}{ Proprietário } & Sagex3 & Datajuri & Senior & Primesoftware \\
\cline { 2 - 5 } & Myrp & Vbbsoftware & Plune & Gestãoclick \\
\cline { 2 - 6 } & e-Agro & Cigam & egestor & Methos \\
\cline { 2 - 6 } & Sispro & QuantoSobra & Desbravador & Bling \\
\cline { 2 - 6 } & ERPFlex & MAXIPROD & SIDICOM & Eccosys \\
\cline { 2 - 6 } & Artsoftsiste & Dataplace & Novi Produção & GMPE \\
\cline { 2 - 6 } & mas & Ausland & Betalabs & ProcelSoftware \\
\cline { 2 - 6 } & Gofitweb & Hmax & Omie & \\
\hline
\end{tabular}

Fonte: Autores (2018)

Dentre os quesitos pesquisados, observados nos Quadros 1 e 2, a maioria deles pode ser respondido com "sim", "não" e "não se sabe". No que tange ao Quadro 2, para softwares livres, a maioria das respostas foi "sim", para os casos em que houve respostas diferenciadas obteve-se os resultados compilados no Quadro 4. Ainda observando os quesitos do Quadro 2, porém agora com foco nos 
softwares gratuitos, apenas o quesito "software adicional?" teve resposta "não" para todos os casos observados, os demais tiveram resposta "sim" para todos os casos, as exceções (respostas que diferiram) estão reunidas no Quadro 5.

Quadro 4-Resposta que diferiram para os softwares livres

\begin{tabular}{|c|c|c|c|c|c|}
\hline \multirow[b]{2}{*}{ Software Livres } & \multicolumn{5}{|c|}{ Respostas que diferiram para os softwares livres } \\
\hline & $\begin{array}{l}\text { Detecção } \\
\text { de erros? }\end{array}$ & $\begin{array}{c}\text { Gerador } \\
\text { de } \\
\text { gráficos? }\end{array}$ & $\begin{array}{l}\text { Possui } \\
\text { Demo? }\end{array}$ & $\begin{array}{c}\text { Mens. } \\
\text { Clara de } \\
\text { erros? }\end{array}$ & $\begin{array}{c}\text { Possui } \\
\text { Integração? }\end{array}$ \\
\hline Fibiese & não & não & $\operatorname{sim}$ & não & não \\
\hline Compiere & $\operatorname{sim}$ & $\operatorname{sim}$ & $\operatorname{sim}$ & $\operatorname{sim}$ & não \\
\hline WebERP & não & não & não & não & não \\
\hline Freedom ERP & não & não & não & não & não \\
\hline Dolibarr ERP & $\operatorname{sim}$ & $\operatorname{sim}$ & $\operatorname{sim}$ & $\operatorname{sim}$ & não \\
\hline Open22ERP & não & $\operatorname{sim}$ & não & não & não \\
\hline OpenXpertya & não & não & $\operatorname{sim}$ & não & não \\
\hline Adempierre ERP & $\operatorname{sim}$ & $\operatorname{sim}$ & $\operatorname{sim}$ & $\operatorname{sim}$ & não \\
\hline ERP CRM & $\operatorname{sim}$ & $\operatorname{sim}$ & $\operatorname{sim}$ & $\operatorname{sim}$ & $\operatorname{sim}$ \\
\hline OpenERP & não & não & não & não & não \\
\hline Vtiger CRM & $\operatorname{sim}$ & Sim & $\operatorname{sim}$ & $\operatorname{sim}$ & não \\
\hline Openbravo & $\operatorname{sim}$ & Sim & $\operatorname{sim}$ & $\operatorname{sim}$ & não \\
\hline
\end{tabular}

Fonte: Autores (2018)

Quadro 5 - Resposta que diferiram para os softwares gratuitos

\begin{tabular}{|c|c|c|}
\hline \multirow{2}{*}{ Software Gratuito } & \multicolumn{2}{|c|}{$\begin{array}{c}\text { Respostas que diferiram para os } \\
\text { softwares gratuitos }\end{array}$} \\
\cline { 2 - 3 } & Possui Demo? & $\begin{array}{c}\text { Gerencia mais de uma } \\
\text { empresa? }\end{array}$ \\
\hline Marketup & sim & sim \\
\hline ERP Lite Free Plus & não & não \\
\hline Bitrix24 Free & não & não \\
\hline Mercado Backoffice & não & não \\
\hline
\end{tabular}

Fonte: Autores (2018)

Observa-se no Quadro 4 que, dos softwares livres identificados, apenas o ERP CRM possui integração com outros sistemas, bem como foi o único que obteve "sim" para todos os quesitos. Nos demais quesitos houve equilíbrio entre respostas "sim" e "não". No que diz respeito aos softwares gratuitos, conforme Quadro 5, apenas o Marketup possui versão de demonstração e permite a gerência de mais de uma empresa.

Quanto aos quesitos observados no Quadro 1, relacionados aos softwares proprietário, obteve-se resposta "não" para todos os casos no quesito "Taxa única?", as demais respostas foram "sim" para todos os casos a exceção das respostas diferenciadas reunidas no Anexo 1.

Os demais quesitos nos Quadros 1 e 2 que não podiam ser respondidos com "sim", "não" e "não se sabe", foram eles: Web site, Sistema Operacional, Indicação de hardware, Número de downloads, Avaliação do autor, Avaliação dos usuários e Dificuldade de instalação. As respostas, referentes aos softwares livres e gratuitos, estão reunidas no Quadro 6. As demais respostas para os softwares proprietários, estão no Anexo 2. 
Quadro 6-Resposta diferiram para os softwares livres e gratuitos

\begin{tabular}{|c|c|c|c|c|c|}
\hline $\begin{array}{l}\text { Software } \\
\text { Livres }\end{array}$ & Web site & $\begin{array}{l}\text { Número de } \\
\text { downloads }\end{array}$ & $\begin{array}{l}\text { Avaliação } \\
\text { do autor }\end{array}$ & $\begin{array}{c}\text { Avaliação } \\
\text { dos } \\
\text { usuários }\end{array}$ & $\begin{array}{c}\text { Dificuldade } \\
\text { de instalação }\end{array}$ \\
\hline Fibiese & sourceforge.net & 0 & Boa & Não há & Alta \\
\hline Compiere & $\begin{array}{c}\text { www.compierbr } \\
\text { asil.com.br }\end{array}$ & 9 & Razoável & Não há & Alta \\
\hline WebERP & $\begin{array}{l}\text { www.weberp.co } \\
\text { m.br }\end{array}$ & 35 & péssima & $\begin{array}{c}4,8 \\
\text { estrelas }\end{array}$ & Alta \\
\hline $\begin{array}{c}\text { Freedom } \\
\text { ERP }\end{array}$ & $\begin{array}{c}\text { www.freedom.o } \\
\text { rg.br }\end{array}$ & $\begin{array}{l}\text { Não se } \\
\text { sabe }\end{array}$ & Boa & Não há & Alta \\
\hline $\begin{array}{c}\text { Dolibarr } \\
\text { ERP }\end{array}$ & $\begin{array}{c}\text { www.dolibarr.co } \\
\text { m.br }\end{array}$ & 4.030 & Boa & $\begin{array}{c}4,5 \\
\text { estrelas }\end{array}$ & Alta \\
\hline $\begin{array}{c}\text { Open22ER } \\
P\end{array}$ & sourceforge.net & 0 & Regular & Não há & Média \\
\hline $\begin{array}{c}\text { OpenXpert } \\
\text { ya }\end{array}$ & sourceforge.net & 6 & Boa & 5 estrelas & Alta \\
\hline $\begin{array}{c}\text { Adempierr } \\
\text { e ERP }\end{array}$ & sourceforge.net & 9 & Razoável & Não há & Alta \\
\hline ERP CRM & sourceforge.net & 10 & Boa & 5 estrelas & Alta \\
\hline OpenERP & $\begin{array}{c}\text { openerpbrasilv } \\
\text { m.sourceforge.n } \\
\text { et }\end{array}$ & 11 & Boa & 5 estrelas & Alta \\
\hline Vtiger CRM & $\begin{array}{c}\text { www.netsac.co } \\
\text { m.br }\end{array}$ & 9 & Boa & Não há & Alta \\
\hline Openbravo & $\begin{array}{c}\text { www.openbravo } \\
\text {.com/pt/ }\end{array}$ & $\begin{array}{l}\text { Não se } \\
\text { sabe }\end{array}$ & Boa & Não há & Alta \\
\hline Marketup & marketup.com & $\begin{array}{c}\text { Não se } \\
\text { sabe }\end{array}$ & Boa & Não há & Baixa \\
\hline $\begin{array}{l}\text { ERP Lite } \\
\text { Free Plus }\end{array}$ & $\begin{array}{c}\text { erplitefree.com. } \\
\text { br }\end{array}$ & 80.000 & Boa & Boa & Baixa \\
\hline $\begin{array}{l}\text { Bitrix24 } \\
\text { Free }\end{array}$ & $\begin{array}{c}\text { www.bitrix24.co } \\
\text { m.br }\end{array}$ & 2.000 .000 & Boa & Boa & Baixa \\
\hline $\begin{array}{l}\text { Mercado } \\
\text { Backoffice }\end{array}$ & $\begin{array}{c}\text { mercadobackoff } \\
\text { ice.com.br }\end{array}$ & +1.000 & Boa & Boa & Baixa \\
\hline
\end{tabular}

Fonte: Autores (2018)

De acordo com os resultados do Quadro 6, os softwares que obtiveram mais downloads foram Bitrix24 Free, seguido de ERP Lite Free Plus, Dolibarr ERP e Mercado Backoffice.

Todos os softwares da modalidade gratuito obtiveram avaliação "Boa" do autor e dos usuários. No que tange à modalidade livre, para aqueles que foram avaliados, a avaliação dos usuários foi boa para todos os softwares. 


\section{CONCLUSÕES}

Esse estudo permitiu mapear softwares de suporte para o PCP, encontrados em língua portuguesa, de forma que suas principais características básicas foram aqui reunidas, fornecendo a comunidade acadêmica e para profissionais que atuam na área, parâmetros de seleção para o uso desses sistemas. Além disso, em virtude das dificuldades típicas encontradas nas micro e pequenas empresas, acredita-se ser de grande contribuição estes resultados. Apesar da amostragem dos softwares não ser tão vasta perante a infinidade de softwares existentes no mercado, apenas 49 softwares, representam ferramentas de suporte para as abordagens MRPI, MRPII e ERP.

No que tange às abordagens, vale ressaltar que, após o mapeamento dos softwares, observou-se que poucos sistemas eram voltados à MRPI e MRPII. Tal fato pode ser explicado em virtude dos usuários procurarem, cada vez mais, sistemas mais robustos que apresentem mais módulos que atendam diversos setores da empresa concomitantemente (não apenas o chão de fábrica). Portanto, a maioria dos softwares encontrados nesta pesquisa, foi voltada para a abordagem ERP. 


\title{
Mapping of production management software: analysis focused on micro and small companies
}

\begin{abstract}
The objective of this research was to obtain a survey of information that could present the academic community and management professionals, software in Portuguese language, based on the MRPI, MRPII and ERP approaches, under the free, free and proprietary modalities, as well as their main functionalities. The research encompasses concepts about Production Planning and Control, Production Management Systems, free software, free and proprietary, advantages and disadvantages in the use of these software, as well as information about micro and small companies and their particularities. The information collected form a three-pronged mapping: feasibility of installation, functionalities and usability, same aspects used in the Campos study (2006). The study was carried out within a qualitative-positivist literature review, a descriptive methodological process in which heuristic and selection analysis was carried out. For the mapping of free software, the site SourceForge.net was used for the free software used related sites and for the owner sites like April, UOL and others. The results showed that there is a greater infinity of ERP software to the detriment of MRPI and MRPII, they also show the general characteristics of the mapped software and other detailed information.
\end{abstract}

KEYWORDS: Production management systems. Free software. Free software. Proprietary software. Micro and small business. 
BARCELOS, I.F. (2001). Aplicação de um processo de usabilidade em uma ferramenta de suporte à escrita técnica em inglês. 126p. Dissertação (Mestrado) - Escola de Engenharia de São Carlos, Universidade de são Paulo, São Carlos, 2001.

BEST SOFTWARE. A complete software evaluation guide for small and mediumsize wholesaler-distributor. Technology Evaluation Centers. Disponível em: <http://simpep.feb.unesp.br>. Acesso em: 30 mar. 2017.

BERALDI, L. C.. Pequena Empresa e Tecnologia da Informação: recomendações e roteiro de aplicação para melhoria da competitividade dos fabricantes de móveis do pólo moveleiro de Mirassol - SP. Tese de Doutorado de Engenharia da Universidade de São Paulo, 2002.

BERALDI, L. C.; FILHO, E. E.. Impacto da Tecnologia de Informação na Gestão de Pequenas Empresas. Revista da Ciência da Informação, Brasília: v. 29, n. 1, p. 4650, jan./abr., 2000.

BOCKERSTETTE, Joseph A., SHELL, Richard L. - "Time Based Manufacturing". New York, McGraw-Hill Inc, 1993, 335 p.

CAMPOS, R,R. Características de sistemas integrados de gestão empresarial desenvolvidos sob o modelo de software livre: informações para suporte a fase de seleção e viabilidade de instalação em pequenas empresas. São Carlos. 2006. Dissertação (Mestrado) - Escola de Engenharia de São Carlos, Universidade de São Paulo,2006. CANALTECH. Software livre é gratuito? Disponível em: $<$ https://canaltech.com.br/coluna/software/O-software-livre-e-gratuito>. Acesso em: 20 de Abril de 2017.

CORRÊA, H. L.; CORRÊA, C. A. Administração de produção e operações: manufatura e serviços: uma abordagem estratégica. 2 ed. São Paulo: Atlas, 2011. 690p. CORRÊA, H.L.; GIANESI, I.G.N.; CAON, M. Planejamento, Programação e Controle da produção. 4a ed. São Paulo: Editora Atlas, 2001. CORRÊA, H.L.; GIANESI, I.G.N.; CAON, M. Planejamento, programação e controle da produção: MRP II/ERP - Conceitos, uso e implantação base para SAP, Oracle Applications e outros softwares integrados de gestão. 5.ed. São Paulo: Atlas, 2011.

FREE SOFTWARE FOUNDATION. O que é software livre. Disponível em: <http://www.gnu.org/philosophy/free-sw.pt-br>. Acesso em: 01 fev. 2017. 
G1. Confiança das micro e pequenas. Disponível em: <g1.globo.com/economia/pme/noticia/2016/08/confianca-de-micro-e-pequenasempresas-e-maior-em-15-meses>. Acesso em: 13 abr. 2017.

G1. Governo anuncia 2 bilhões em financiamento para micro e pequenas empresas. Disponível em: <http://g1.globo.com/politica/noticia/governoanuncia-r-82-bilhoes-em-financiamentos-para-pequenas-emicroempresas.ghtml>. Acesso em: 13 abr. 2017.

GUARDA, C. N. M. Características econômicas das micro e pequenas empresas industriais. Disponível em: <https://www.gestiopolis.com/caracteristicaseconomicas-das-micro-e-pequenas-empresas-industriais/>. Acesso em: 29 abr. 2017.

MARTINS, G.A. (1994). Epistemologia da pesquisa em administração. Tese (Livre docência) - Faculdade de Economia, Administração e Contabilidade, Universidade de São Paulo, São Paulo, 1995.

MARTINS; LAUGENI. Administração da Produção. São Paulo . Saraiva. 2015.

MORAES, G. D. A.. Tecnologia da Informação na Pequena Empresa: uma investigação sobre sua contribuição à gestão estratégica da informação em empreendimentos industriais dos minidistritos de São José do Rio Preto-SP. Dissertação de mestrado da Faculdade de Engenharia de São Paulo, 2005.

REIS, C.R. (2003). Caracterização de um processo de software para projetos de software livre. 158p. Dissertação (Mestrado) - Escola de Engenharia de São Carlos, Universidade de São Paulo, São Carlos, 2003.

SLACK,N,CHAMBERS,S; JOHNSTON,R.Administração da produção.4o ed.São Paulo.Atlas,2015.

SEBRAE. Vantagens de se usar software livre. Disponível em: <https://www.sebrae.com.br/sites/portalsebrae/artigos/o-que-e-software-livree-quais-as-vantagens-em-usa-lo-na-sua-empresa>. Acesso em: 26 abr. 2017.

SEBRAE.Serviço Brasileiro de Apoio às Micro e Pequenas Empresas, Sebrae 2014 .Disponível em: <//www.sebrae.com.br/sites/PortalSebrae/ufs/mt/noticias/micro-e-pequenasempresas-geram-27-do-pib-do-brasil >. Acesso em: 15 de Fevereiro de 2017.

SILVEIRA, SÉRGIO AMADEU DA. Software livre: a luta pela liberdade do conhecimento.São Paulo : Editora Fundação Perseu Abramo, 2004 
TUBINO, D. F. Planejamento e Controle da Produção: Teoria e Prática. São Paulo: Atlas, 2009.

TOSTES, LUÍS FERNADES ROGÉRIO. Análise da implementação de sistemas ERP em pequenas empresas auxiliadas por software livre. São Carlos, 2009.

UOL. Sebrae aposta que 2017 será o ano das micro e pequenas empresas. Disponível em:<http://jcrs.uol.com.br/_conteudo/2017/01/ge/noticias/540913sebrae-aposta-que-2017-sera-o-ano-das-micro-e-pequenas-empresas.html>. Acesso em: $21 \mathrm{abr}$.

Recebido: 14 Nov. 2018

Aprovado: 06 Out. 2020

DOI: 10.3895/gi.v16n1.9055

Como citar:

MENDES JUNIOR, L.C. et al. Mapeamento de softwares de gestão da produção: análise voltada para as micro e pequenas empresas. R. Gest. Industr., Ponta Grossa, v. 16, n. 1, p. 01-21, Jan./Mar. 2020.

Disponível em: http://periodicos.utfpr.edu.br/revistagi

Correspondência:

Luís Cláudio Figueira Mendes Júnior

Universidade do Estado do Pará (UEPA), Castanhal, Pará, Brasil.

Direito autoral: Este artigo está licenciado sob os termos da Licença Creative Commons-Atribuição 4.0

Internacional.

(c) (1) 
ANEXO 1 - Resposta que diferem para os softwares proprietário

\begin{tabular}{|c|c|c|c|c|c|}
\hline \multirow{2}{*}{$\begin{array}{c}\text { Software } \\
\text { Proprietário }\end{array}$} & \multicolumn{5}{|c|}{ Respostas que diferiram } \\
\hline & Detecção de erros? & Gerador de gráficos? & Possui Demo? & Mens. Clara de erros? & Possui Integração? \\
\hline Sagex3 & não & $\operatorname{sim}$ & Sim & não & não \\
\hline Myrp & não & $\operatorname{sim}$ & Sim & não & não \\
\hline e-Agro & não & $\operatorname{sim}$ & Sim & não & não \\
\hline Sispro & $\operatorname{sim}$ & $\operatorname{sim}$ & Sim & $\operatorname{sim}$ & não \\
\hline ERPFlex & $\operatorname{sim}$ & $\operatorname{sim}$ & Não & $\operatorname{sim}$ & não \\
\hline Gestactus & $\operatorname{sim}$ & $\operatorname{sim}$ & Sim & $\operatorname{sim}$ & $\operatorname{sim}$ \\
\hline Artsoftsistemas & não & $\operatorname{sim}$ & Sim & não & não \\
\hline Gofitweb & não & $\operatorname{sim}$ & Sim & não & $\operatorname{sim}$ \\
\hline Datajuri & não & $\operatorname{sim}$ & Sim & não & não \\
\hline Vbbsoftware & não & $\operatorname{sim}$ & Sim & não & não \\
\hline Cigam & não & $\operatorname{sim}$ & Sim & não & não \\
\hline QuantoSobra & sim & sim & Sim & $\operatorname{sim}$ & não \\
\hline MAXIPROD & $\operatorname{sim}$ & $\operatorname{sim}$ & Sim & $\operatorname{sim}$ & não \\
\hline Dataplace & não & $\operatorname{sim}$ & Sim & não & não \\
\hline Ausland & não & $\operatorname{sim}$ & Sim & não & não \\
\hline Hmax & não & $\operatorname{sim}$ & Sim & não & não \\
\hline Senior & não & $\operatorname{sim}$ & Sim & não & não \\
\hline Plune & não & $\operatorname{sim}$ & Sim & não & não \\
\hline Egestor & não & $\operatorname{sim}$ & Sim & não & não \\
\hline Desbravador & não se sabe & $\operatorname{sim}$ & Não & não se sabe & não se sabe \\
\hline SIDICOM & $\operatorname{sim}$ & $\operatorname{sim}$ & Sim & $\operatorname{sim}$ & não \\
\hline Novi Produção & $\operatorname{sim}$ & $\operatorname{sim}$ & Sim & $\operatorname{sim}$ & $\operatorname{sim}$ \\
\hline Betalabs & $\operatorname{sim}$ & $\operatorname{sim}$ & Sim & $\operatorname{sim}$ & não \\
\hline Omie & não & $\operatorname{sim}$ & Sim & não & não \\
\hline Primesoftware & não & sim & não & não & não \\
\hline Gestaoclick & não & $\operatorname{sim}$ & $\operatorname{sim}$ & não & não \\
\hline Methos & não & $\operatorname{sim}$ & $\operatorname{sim}$ & não & não \\
\hline Bling & não & $\operatorname{sim}$ & $\operatorname{sim}$ & não & $\operatorname{sim}$ \\
\hline Eccosys & $\operatorname{sim}$ & $\operatorname{sim}$ & $\operatorname{sim}$ & $\operatorname{sim}$ & $\operatorname{sim}$ \\
\hline GMPE & não & $\operatorname{sim}$ & $\operatorname{sim}$ & não & não \\
\hline ProcelSoftware & $\operatorname{sim}$ & $\operatorname{sim}$ & $\operatorname{sim}$ & $\operatorname{sim}$ & não \\
\hline
\end{tabular}

Fonte (autores 2018) 
ANEXO 2 - Demais respostas para os softwares proprietário

\begin{tabular}{|c|c|c|c|c|c|}
\hline $\begin{array}{c}\text { Software } \\
\text { Proprietário }\end{array}$ & Web site & Número de downloads & Avaliação do autor & Avaliação dos usuários & $\begin{array}{l}\text { Dificuldade de } \\
\text { instalação }\end{array}$ \\
\hline Sagex3 & www.sagex3.com & Não se sabe & Boa & Boa & Baixa \\
\hline Myrp & www.myrp.com.br & Não se sabe & Boa & Boa & Baixa \\
\hline e-Agro & eagroweb.com & Não se sabe & Boa & Boa & Baixa \\
\hline Sispro & www.sispro.com.br & Não se sabe & Boa & Boa & Baixa \\
\hline ERPFlex & lps.erpflex.com.br & +2000 & Boa & Boa & Baixa \\
\hline Gestactus & www.exactus.com.br & Não se sabe & Boa & Boa & Baixa \\
\hline Gofitweb & gofitweb.com & Não se sabe & Boa & Boa & Baixa \\
\hline Datajuri & www.datajuri.com.br & Não se sabe & Boa & Boa & Baixa \\
\hline Vbbsoftware & www.vbbsoftware.com & Não se sabe & Boa & Boa & Baixa \\
\hline Cigam & www.cigam.com.br & 5.000 & Boa & Boa & Baixa \\
\hline QuantoSobra & www.quantosobra.com.br & Não se sabe & Boa & Boa & Baixa \\
\hline MAXIPROD & www.maxiprod.com.br & Não se sabe & Boa & Boa & Baixa \\
\hline Dataplace & www.dataplace.com.br & Não se sabe & Boa & Boa & Baixa \\
\hline Ausland & ausland.com.br & Não se sabe & Boa & Boa & Baixa \\
\hline Senior & www.senior.com.br & Não se sabe & Boa & Boa & Baixa \\
\hline Plune & www.plune.com.br & Não se sabe & Boa & Boa & Baixa \\
\hline Egestor & www.egestor.com.br & Não se sabe & Boa & Boa & Baixa \\
\hline Desbravador & www.desbravador.com.br & Não se sabe & Regular & Boa & Não se sabe \\
\hline SIDICOM & sidicom.com.br & Não se sabe & Boa & Boa & Baixa \\
\hline Novi Produção & Www.novisistemas.com.br & Não se sabe & Boa & Boa & Baixa \\
\hline Betalabs & betalabs.com.br & Não se sabe & Boa & Boa & Baixa \\
\hline Omie & www.omie.com.br & Não se sabe & Boa & Boa & Baixa \\
\hline Primesoftware & primesoftware.com.br & Não se sabe & Boa & Boa & Baixa \\
\hline Gestaoclick & gestaoclick.com.br & Não se sabe & Boa & Boa & Baixa \\
\hline Methos & www.methos.com.br & Não se sabe & Boa & Boa & Baixa \\
\hline Bling & www.bling.com.br & Não se sabe & Boa & Boa & Baixa \\
\hline Eccosys & www.eccosys.com.br & Não se sabe & Boa & Boa & Baixa \\
\hline GMPE & gmpe.com.br & Não se sabe & Boa & Boa & Baixa \\
\hline ProcelSoftware & www.procelsoftware.com.br & Não se sabe & Boa & Boa & Baixa \\
\hline
\end{tabular}

Fonte (autores 2018) 